OPEN ACCESS

Edited by:

Ovidiu Constantin Baltatu Anhembi Morumbi University, Brazil

Reviewed by:

Paul Kenneth Witting

University of Sydney, Australia

Charles Dudley Mills,

BioMedical Consultants, USA

*Correspondence:

Xiu-Fen Ming

xiu-fen.ming@unifr.ch

Zhihong Yang

zhihong.yang@unifr.ch

${ }^{\dagger}$ These authors have contributed equally to this work.

Specialty section:

This article was submitted to Integrative Physiology,

a section of the journa

Frontiers in Physiology

Received: 30 June 2016 Accepted: 04 November 2016 Published: 22 November 2016

Citation:

Huang J, Rajapakse A, Xiong Y, Montani J-P, Verrey $F$, Ming $X$ - $F$ and Yang Z (2016) Genetic Targeting of Arginase-Il in Mouse Prevents Renal Oxidative Stress and Inflammation in

Diet-Induced Obesity.

Front. Physiol. 7:560.

doi: 10.3389/fphys.2016.00560

\section{Genetic Targeting of Arginase-II in Mouse Prevents Renal Oxidative Stress and Inflammation in Diet-Induced Obesity}

\author{
Ji Huang ${ }^{1,2+}$, Angana Rajapakse ${ }^{1 \dagger}$, Yuyan Xiong ${ }^{1}$, Jean-Pierre Montani ${ }^{1,2}$, \\ François Verrey ${ }^{2,3}$, Xiu-Fen Ming ${ }^{1,2 *}$ and Zhihong Yang ${ }^{1,2 *}$ \\ ${ }^{1}$ Cardiovascular and Aging Research, Division of Physiology, Department of Medicine, University of Fribourg, Fribourg, \\ Switzerland, " Swiss National Centre of Competence in Research (NCCR) Kidney Control of Homeostasis "Kidney.CH", \\ Zurich, Switzerland, ${ }^{3}$ Institute of Physiology, University of Zurich, Zurich, Switzerland
}

Obesity is associated with development and progression of chronic kidney disease (CKD). Recent evidence demonstrates that enhanced levels of the L-arginine:ureahydrolase, including the two isoenzymes arginase-I (Arg-I) and arginase-II (Arg-II) in vascular endothelial cells promote uncoupling of endothelial nitric oxide synthase (eNOS), leading to increased superoxide radical anion and decreased NO production thereby endothelial dysfunction. Arg-II but not Arg-l is abundantly expressed in kidney and the role of Arg-II in CKD is uncertain and controversial. We aimed to investigate the role of Arg-II in renal damage associated with diet-induced obesity mouse model. Wild type (WT) C57BL/6 mice and mice deficient in Arg-II gene (Arg- $\mathrm{II}^{-/-}$) were fed with either a normal chow (NC) or a high-fat-diet (HFD) for 14 weeks (starting at the age of 7 weeks) to induce obesity. In WT mice, HFD feeding caused frequent renal lipid accumulation, enhancement of renal reactive oxygen species (ROS) levels which could be attenuated by a NOS inhibitor, suggesting uncoupling of NOS in kidney. HFD feeding also significantly augmented renal Arg-ll expression and activity. All the alterations in the kidney under HFD feeding were reduced in Arg-II-/- mice. Moreover, mesangial expansion as analyzed by Periodic Acid Schiff (PAS) staining and renal expression of vascular adhesion molecule-1 (VCAM-1) and intercellular adhesion molecule-1 (ICAM-1) in HFD-fed WT mouse assessed by immunoblotting were reduced in the HFD-fed Arg-II-/- mice, although there was no significant difference in body weight and renal weight/body weight ratio between the WT and Arg- $\|^{-/-}$mice. Thus, Arg-II expression/activity is enhanced in kidney of diet-induced obesity mice. Genetic targeting of Arg-II prevents renal damage associated with obesity, suggesting an important role of Arg-II in obesity-associated renal disease development.

\footnotetext{
Keywords: adhesion molecules, arginase, oxidative stress, obesity, renal disease
} 


\section{INTRODUCTION}

According to the latest Non-Communicable Diseases RiskFactor Collaboration (NCD-RisC) report, in 2014 there were 422 million people worldwide who had diabetes, i.e., roughly a four-fold increase over the past 35 years (Collaboration, 2016). Diabetic nephropathy or diabetic chronic kidney disease (CKD) is one of the important diabetic complications representing the most common cause of end-stage renal disease (Collins et al., 2013). Although the mechanisms underlying obesity-associated diabetic renal damage are complex and have not yet been fully understood, it has been demonstrated that ectopic renal lipid accumulation, inflammation including macrophage infiltration in kidney through adhesion molecules, i.e., vascular adhesion molecule-1 (VCAM-1) and intercellular adhesion molecule1 (ICAM-1), glomerulus mesangial expansion, impaired endothelial nitric oxide (NO) bioavailability and oxidative stress or increased ROS generation in kidney are importantly involved in the development of diabetic renal damage (Nakagawa et al., 2011).

There are studies showing key roles of endothelial nitric oxide synthase (eNOS) in protection against development of diabetic renal disease (Wang et al., 2011; Cheng et al., 2012; Cheng and Harris, 2014). Functional alteration of eNOS, in particular, eNOS-uncoupling, a situation that eNOS enzyme produces more superoxide radical anion instead of NO, has been shown to be critical in endothelial dysfunction and diabetic renal disease (Goligorsky et al., 2001). Under eNOS-uncoupling condition, superoxide radical anion reacts with $\mathrm{NO}$, resulting in decreased NO bioavailability and increased generation of the more potent pro-oxidant peroxynitrite causing nitrosative stress (Förstermann and Sessa, 2012). NO is derived from L-arginine
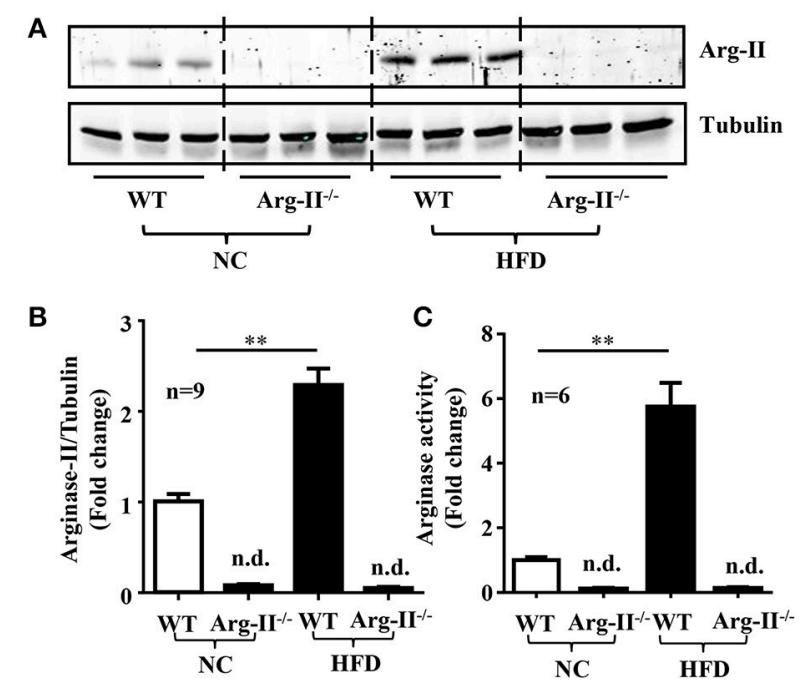

FIGURE 1 | Enhanced renal Arg-II expression and activity in HFD-induced obesity. (A) Immunoblotting analysis of Arg-II expression in WT and Arg- $\|^{-/}$mice with NC and HFD. (B) Bar graphs show quantification of Arg-Il protein expression and (C) activity. $n=9$ for protein levels and $n=6$ for enzymatic activity, ${ }^{\star *} p<0.01$ between indicated groups, n.d., not detectable. and decrease in $\mathrm{NO}$ is not only important for pathogenesis of vascular complications but also for progression of renal disease associated with diabetes (Cheng and Harris, 2014). Among other mechanisms, elevated activity and/or expression of arginase i.e., the L-arginine:ureahydrolase including arginase-I (Arg-I) and arginase-II (Arg-II) isoenzyme, has been shown to cause eNOSuncoupling, resulting in increased oxidative stress in aging and age-associated cardiovascular diseases including atherosclerosis and type 2 diabetic vascular dysfunctions (Yepuri et al., 2012; Li and Förstermann, 2013).

Arg-I and Arg-II are encoded by two separate genes (Sparkes et al., 1986; Gotoh et al., 1997). Arg-I is located in cytosol, while Arg-II is in mitochondrion (Ash, 2004). Despite different subcellular localization, the two isoenzymes share the same substrate L-arginine and compete with eNOS for L-arginine, thereby decreasing intracellular L-arginine bioavailability for production of NO (Yang and Ming, 2013). Under the condition of L-arginine deficiency caused by elevation of either Arg-I or Arg-II, eNOS becomes uncoupled, leading to oxidative stress and endothelial dysfunction in cardiovascular diseases and aging ( $\mathrm{Li}$ and Förstermann, 2013; Yang and Ming, 2013). Hence, eNOSuncoupling has been shown to be an important mechanism for oxidative stress in cardiovascular pathology.

It is well known that Arg-I is mainly expressed in hepatocytes and is an essential enzyme involved in hepatic urea cycle for detoxification of ammonia (Crombez and Cederbaum, 2005; Tsang et al., 2012). In contrast, Arg-II is highly expressed in kidney (Yang and Ming, 2014). The function of Arg-II in kidney is, however, less well defined. There is only a limited number of studies indicating that Arg-II is upregulated and is detrimental for renal function in type 1 diabetes animal models (Morris et al., 2011; Toque et al., 2013; You et al., 2013, 2014). However, whether Arg-II is also involved in obesity-associated type 2 diabetic renal disease is not known. In contrast to these studies, a recent study suggests that lack of Arg-II even accelerates some of the diabetes-induced renal damages in type 1 diabetic mouse model (Romero et al., 2013).

The aim of our study is to investigate whether Arg-II is involved in renal damage in a diet-induced obesity mouse model.

\section{MATERIALS AND METHODS}

\section{Materials}

Antibodies against Arg-II (sc-20151), ICAM-1(sc-8439), and VCAM-1 (sc-8304) were purchased from Santa Cruz Technology Inc. (Dallas, USA); Antibody against tubulin (T5168), 3nitrotyrosine (06-284) and Periodic Acid-Schiff (PAS) staining kit (395B) were from Sigma (Buchs, Switzerland); Antibody against B0AT3 (Slc6a18) was produced and validated as previously described (Romeo et al., 2006). IRDye800-conjugated antirabbit IgG (926-32211) was purchased from LI-COR Biosciences (Lincoln, USA); Alexa Fluor 680-conjugated anti-mouse IgG (A21057), Alexa Fluor 488 conjugated-Goat anti-rabbit IgG $(\mathrm{H}+\mathrm{L})$ secondary antibody (A-11008) and dihydroethidium (DHE, D23107) were from Invitrogen/Thermo Fisher Scientific (Waltham, MA USA). 


\section{Animal Models}

Arg-II ${ }^{-/}-$mice were kindly provided by Dr. William O'Brien (Shi et al., 2001) and backcrossed to C57BL/6J for more than 10 generations (Ming et al., 2012). Genotyping was performed by polymerase chain reaction (PCR) as previously described (Shi et al., 2001). The WT and Arg-II ${ }^{-/-}$offsprings from hetero/hetero cross were interbred to obtain WT and Arg$\mathrm{II}^{-/-}$mice, respectively, for experiments. Starting at the age of 7 weeks, the male WT and Arg- $\mathrm{II}^{-/-}$mice were given free access during 14 weeks to either a normal chow (NC; energy content: $10.6 \%$ fat, $27.6 \%$ protein, and $57 \%$ carbohydrate, fiber 4.8\%; Provimi Kliba NAFAG 3436; Kaiseraugst, Switzerland) or a high fat diet (HFD, energy content: $55 \%$ fat, $21 \%$ protein, and 24\% carbohydrate; Harlan Teklad TD 93075; Horst, Netherlands). Animals were sacrificed after 14 weeks of HFD feeding. The kidneys were collected for histological analysis or snap-frozen in liquid nitrogen and kept at $-80^{\circ} \mathrm{C}$ until used for immunoblotting analysis and arginase activity assay or

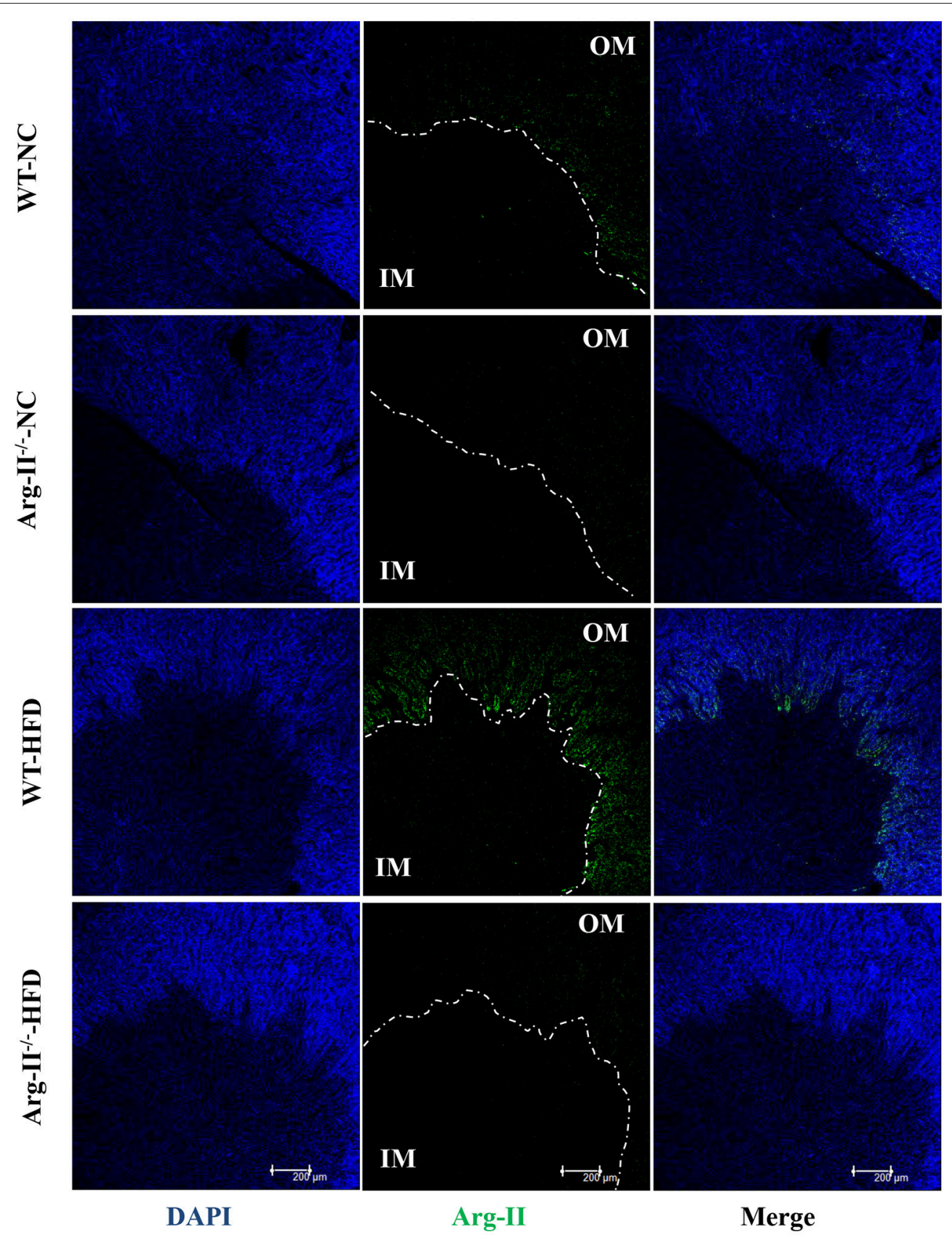

FIGURE 2 | Arg-II is expressed in outer medulla of kidney. Confocal immunofluorescence staining of Arg-II (green) and DAPI (blue) in the kidney of WT and Arg- $\|^{-/}-$mice fed either a normal chow (NC) or high fat diet (HFD). Representative images of individual staining and merged images are shown $(n=4$ for each group). OM, outer medulla; IM, inner medulla. 
cryosectioning. Due to the fact that not all the experiments can be done with the same kidney samples for different purposes such as immunoblotting, enzymatic activity assay, oxidative/nitrosative stress measurement, and immunofluorescence staining, PAS staining, etc., various series of experiments with mice on NC and HFD were initiated. The protocol of animal handling and experimentation was approved by the Service de la sécurité alimentaire et des affaires vétérinaires, Etat de Fribourg, Switzerland.

\section{Oil Red O Staining}

Cryosections of kidneys ( $7 \mu \mathrm{m}$ thick) were fixed with $4 \%$ paraformaldehyde for 10 minutes followed by briefly washing with running tap water. After quickly rinsing with $60 \%$ isopropanol, the sections were stained with freshly prepared and filtered Oil Red $\mathrm{O}$ working solution for 15 minutes, followed by rinsing with $60 \%$ isopropanol and washing with distilled water (Ramírez-Zacarías et al., 1992). Images were obtained with Zeiss microscope.

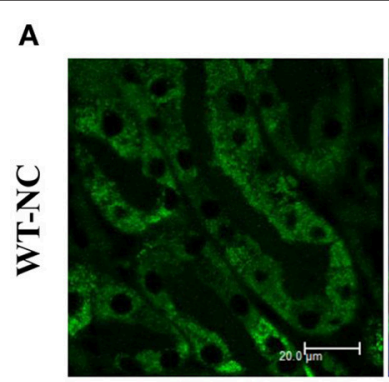

Arg-II

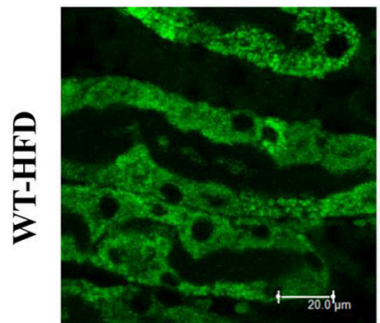

Arg-II

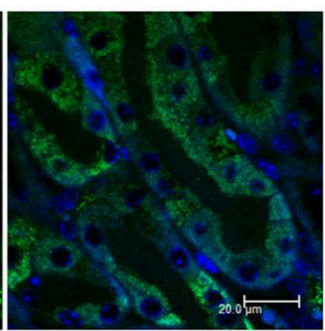

Merge

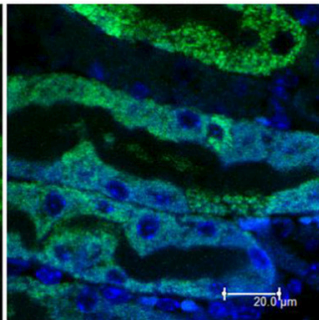

Merge

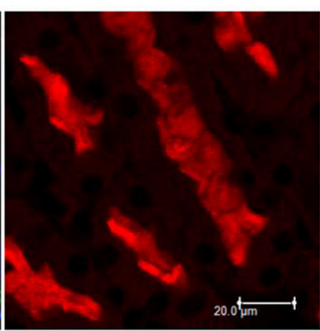

B0AT3

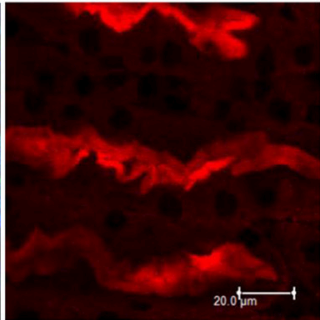

B0AT3

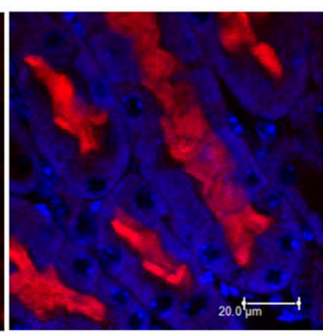

Merge

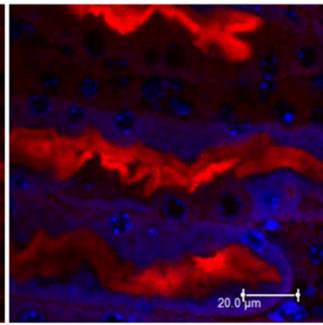

Merge

B

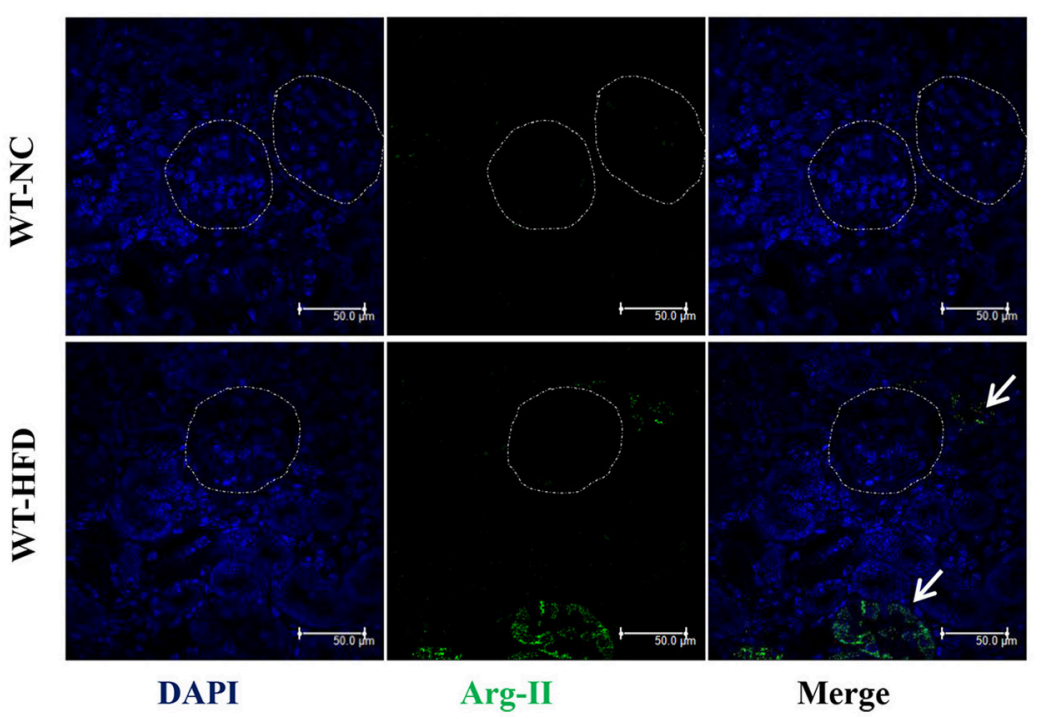

FIGURE 3 | Arg-II is localized in proximal straight tubules. (A) Confocal immunofluorescence staining in consecutive sections demonstrates that Arg-II (green) is localized in the S3 proximal straight tubules which are positive for BOAT3 (Slc6a18, red, a luminal marker of S3 proximal straight tubules) in WT mice fed either a normal chow (NC) or high fat diet (HFD). (B) No obvious Arg-II (green) signal was detected in glomeruli (circled area) in WT mice fed either NC or HFD. Some Arg-II signals were observed in tubules outside glomeruli (green indicated by arrows). Representative images of individual staining and merged images are shown $(n=4)$. 


\section{Mesangial Expansion}

3- $\mu \mathrm{m}$ paraffin sections of kidneys were stained with periodic acid Schiff (PAS) staining kit according to manufacturer's instruction. All images were obtained with Zeiss microscope. The mesangial expansion was analyzed by percentage of PAS-positive material which occupies the mesangial matrix within the tuft. A minimum of 20 glomeruli in each specimen was examined.

\section{Kidney Arginase Activity Assay}

Arginase activity in kidney lysates was measured by colorimetric determination of urea formed from L-arginine in an in vitro activity assay as previously described (Xiong et al., 2013). Briefly, kidney powders were lysed in lysis buffer containing $10 \mathrm{mmol} / \mathrm{L}$ Tris- $\mathrm{HCl}(\mathrm{pH} 7.4), 0.4 \%$ Triton $\mathrm{X}-100,10 \mu \mathrm{g} / \mathrm{mL}$ leupeptin, and $0.1 \mathrm{mmol} / \mathrm{L}$ phenylmethylsulfonyl fluoride (PMSF). Samples were centrifuged at $16,200 \times \mathrm{g}$ at $4^{\circ} \mathrm{C}$ for $10 \mathrm{~min}$, and protein concentration of the supernatant was determined by the Lowry method (Bio-Rad). For measurement of arginase activity, an equal amount of the cell lysate was added to $50 \mu \mathrm{L}$ of Tris- $\mathrm{HCl}$ ( $10 \mathrm{mmol} / \mathrm{L}[\mathrm{pH} 7.4]$ ) containing $5 \mathrm{mmol} / \mathrm{L} \mathrm{MnCl}_{2}$. Arginase was then activated by heating the mixture at $56^{\circ} \mathrm{C}$ for $10 \mathrm{~min}$. The hydrolysis reaction of L-arginine by arginase was conducted by incubating the mixture containing activated arginase with $100 \mu \mathrm{L}$ of L-arginine $(100 \mathrm{mmol} / \mathrm{L}[\mathrm{pH} \mathrm{9.6}])$ at $37^{\circ} \mathrm{C}$ for $1 \mathrm{~h}$. For colorimetric determination of urea, $1 \mathrm{~mL}$ of chromogenic reagent consisting of 1 volume of 3\% 2,3-butanedione monoxine and 29 volumes of the acid solution mixture $\left(\mathrm{H}_{2} \mathrm{SO}_{4}: \mathrm{H}_{3} \mathrm{PO}_{4}: \mathrm{H}_{2} \mathrm{O}\right.$ 1:3:7) was added, and the mixture was then heated at $100^{\circ} \mathrm{C}$ for $30 \mathrm{~min}$. After placing the samples in the dark for $10 \mathrm{~min}$
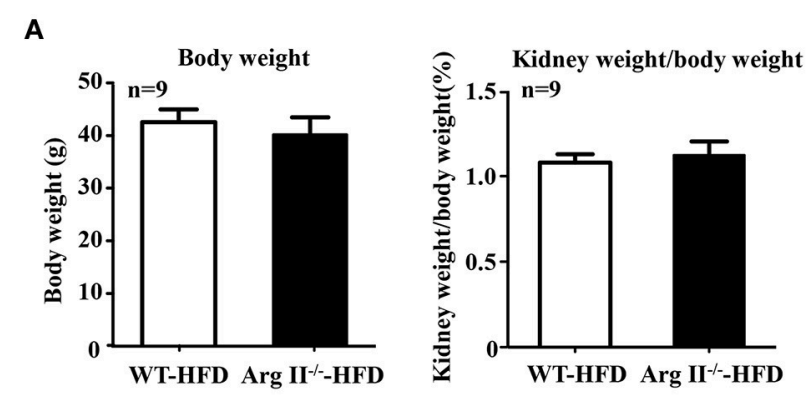

B

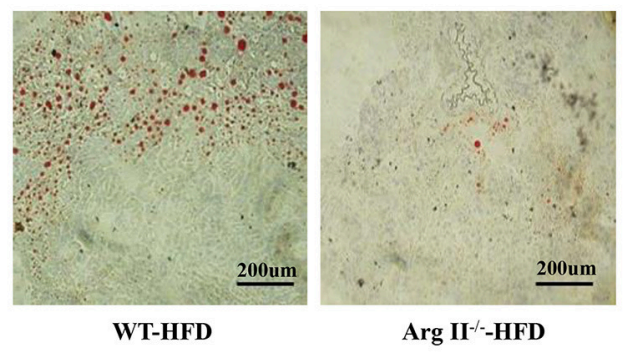

FIGURE 4 | Body weight, kidney weight/body weight ratio, and renal lipid accumulation in obesity. Starting at the age of 7 weeks, WT and Arg- $\|^{-/}$mice were fed with HFD for 14 weeks. (A) Body weight and ratio of kidney weight/body weight after 14 weeks HFD; $n=11$ for WT mice, $n=9$ for Arg- $\|^{-/}-$mice. (B) Representative images of Oil Red $O$ staining of renal section. $n=9$ /group. at room temperature, the urea concentration was determined spectrophotometrically by the absorbance at $492 \mathrm{~nm}$. The amount of the urea produced was used as an index for arginase activity.

\section{Immunoblotting}

Tissue lysate preparation, SDS-PAGE, and transfer of SDS gels to Immobilon-P membranes (Millipore) were performed as previously described (Ming et al., 2012). The resultant membrane was first incubated with the corresponding primary antibody overnight at $4^{\circ} \mathrm{C}$ with gentle agitation after blocking with 5\% skim milk. After washing, the blot was then further incubated with corresponding anti-mouse (Alexa Fluor 680-conjugated) or anti-rabbit (IRDye 800conjugated) secondary antibodies. Signals were visualized using the Odyssey Infrared Imaging System (LI-COR Biosciences, USA). Quantification of the signals was performed using NIH Image 1.62 software (US National Institutes of Health).

\section{Confocal Immunofluorescence Staining of Arg-II and 3-NT}

Kidneys from the WT and Arg- $\mathrm{II}^{-/-}$mice were isolated and fixed with $3.7 \%$ paraformaldehyde and embedded in paraffin. After deparaffinization in xylene, hydration in ethanol, and antigen retrieval in Tris-EDTA buffer $(10 \mathrm{mmol}$ Tris Base, $1 \mathrm{mM}$ EDTA, 0.05\% Tween 20, pH 9.0) in a pressure cooker, paraffin-embedded sections $(5 \mu \mathrm{m})$ were blocked with $1 \% \mathrm{BSA}$ in PBS for $60 \mathrm{~min}$ and were then incubated with the first antibodies (Arg-II 1:100, B0AT3 1:200, 3 nitrotyrosine $1: 200$ ) at $4^{\circ} \mathrm{C}$ overnight and subsequently with fluorescence-labeled secondary antibodies at room temperature for $2 \mathrm{~h}$, followed by counterstaining with $300 \mathrm{nmol} / \mathrm{L}$ DAPI (4'6-diamidino-2-phenyl-indole-dihydrochloride, Invitrogen) for $3 \mathrm{~min}$. The immunofluorescence signals were visualized under LEICA's DIM6000 Confocal microscope. The intensity of the fluorescence was quantified by NIH Image $\mathrm{J}$ software and expressed as intensity per arbitrary unit of area.

\section{Detection of ROS}

Detection of ROS levels was performed as described previously (Rajapakse et al., 2011). For measurement of ROS, pretreated without or with NOS inhibitor L-NAME $(1 \mathrm{mmol} / \mathrm{L}$, $1 \mathrm{~h}$ ), cryosections were incubated with $5 \mu \mathrm{mol} / \mathrm{L}$ DHE in PBS for 30 minutes. Samples were then washed 3 times and images were obtained with Zeiss fluorescence microscope. The intensity of the fluorescence was quantified by NIH Image J software.

\section{Statistics}

Data are given as mean \pm SEM. In all experiments, $n$ represents the number of experiments or animals. Statistical analysis was performed with Student's unpaired $t$-test or analysis of variance (ANOVA) with Tukey-test. Differences in mean values were considered significant at two tailed $P \leq 0.05$. 


\section{RESULTS}

\section{Increased Arg-II Expression/Activity in HFD-Induced Obesity}

In the WT mice, HFD feeding significantly enhanced ArgII expression and arginase activity in kidney (Figure 1). As expected, no Arg-II expression and arginase activity could be detected in $\mathrm{Arg}-\mathrm{II}^{-/-}$mouse kidney, demonstrating that renal arginase activity is attributable to Arg-II isoenzyme. Confocal microscopic immunofluorescence staining showed that ArgII was mainly expressed in tubules of outer medulla, which is increased in the HFD fed WT mice (Figure 2). Further experiments in consecutive sections demonstrated that Arg-II is expressed in the tubule cells which are positive for B0AT3 (Slc6a18), a luminal marker of S3 straight segment of proximal tubules (Figure 3A). No significant signal of Arg-II in the glomeruli could be detected either in mice fed NC or HFD (Figure 3B).

\section{Arg-II-Deficiency Protects Against Ectopic Renal Lipid Accumulation in Obesity}

No difference in body weight and kidney weight/body weight ratio was observed between $\mathrm{WT}$ and $\mathrm{Arg}-\mathrm{II}^{-/-}$mice fed HFD (Figure 4A). Lipid accumulation in kidney was frequently observed in the obese WT mice, whereas this was not the case in the Arg-II ${ }^{-/}$mice (Figure 4B). The results indicate that ArgII deficiency protects against ectopic renal lipid accumulation in diet-induced obesity.

\section{Arg-II-Deficiency Prevents Renal Oxidative Oxygen Species (ROS) Generation, Adhesion Molecule Expression and Glomerulus Mesangial Expansion}

In the WT mice fed HFD, there was a significant higher level of ROS generation (DHE staining) in the glomeruli as compared to the Arg-II ${ }^{-/}$mice on HFD (Figure 5). Interestingly, the ROS generation was inhibited by the NOS inhibitor L-NAME $\left(10^{-3}\right.$ $\mathrm{mol} / \mathrm{L}$, Figure 5), demonstrating NOS-uncoupling in obesity kidney. Similarly, peroxynitrite levels as demonstrated by 3-NT staining were increased in HFD fed WT mice and this obesityinduced increase in peroxynitrite was prevented in the Arg- $\mathrm{II}^{-/-}$ mice (Figure 6). Moreover, Arg- $\mathrm{II}^{-/-}$mice revealed decreased mesangial expansion (Figure 7) and lower levels of renal ICAM-1 and VCAM-1 as demonstrated by immunoblotting (Figure 8).

\section{DISCUSSION}

In this study, we demonstrate that HFD-induced obesity is associated with renal injury markers, i.e., oxidative stress, as assessed by increased DHE staining and peroxynitrite levels, enhanced levels of inflammatory adhesion molecules, mesangial expansion, and more frequent lipid accumulation in kidney, which are characteristics of diabetic kidney disease as demonstrated by several studies in the same animal model (Lal et al., 2000; DeRubertis et al., 2004; Menini et al., 2006; Fioretto et al., 2008; Deji et al., 2009; Declèves et al., 2011; Cui et al., 2013).

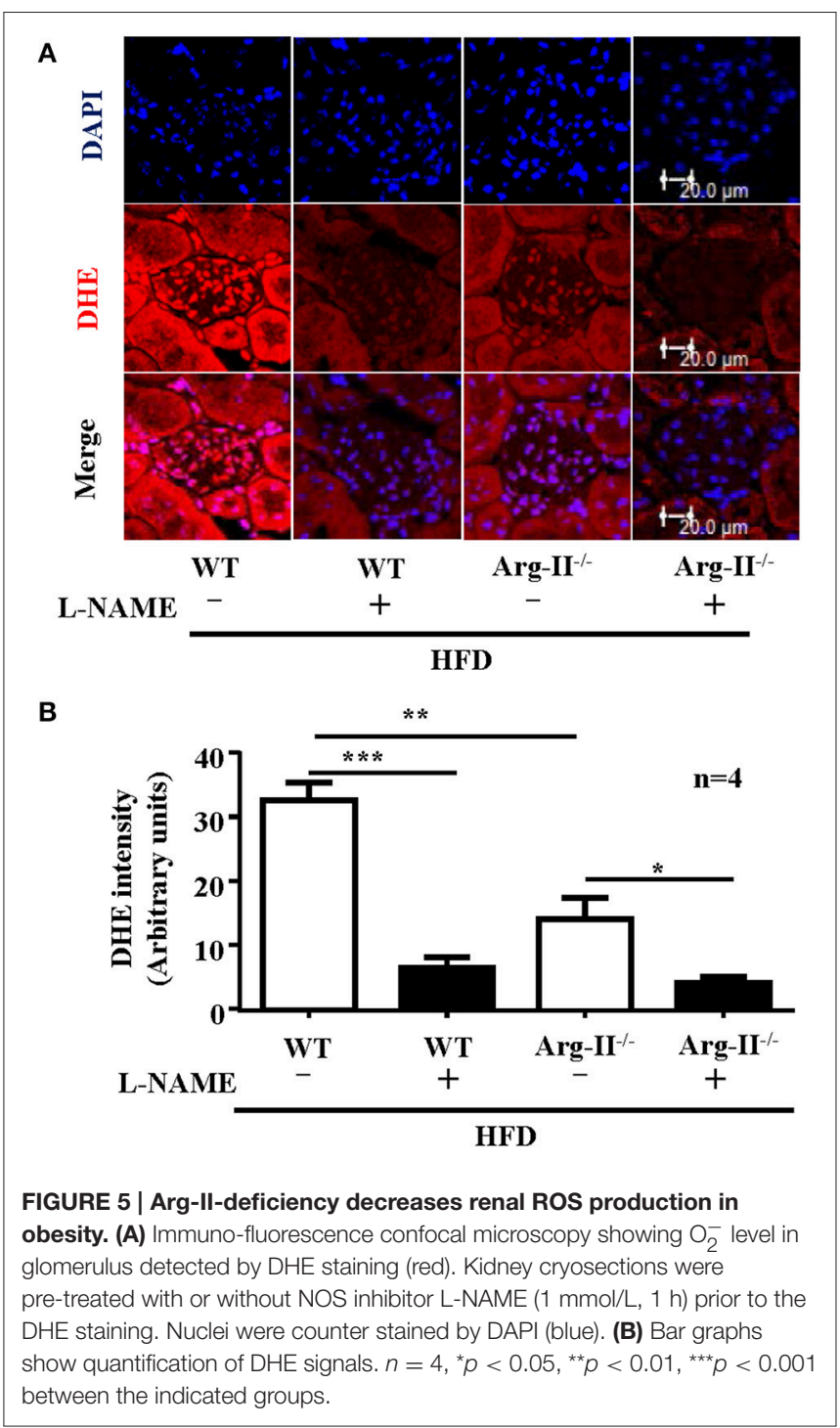

These renal alterations are important mechanisms leading to end stage renal disease (ESRD) associated with obesity. Importantly, our current study provides novel evidence demonstrating that diet-induced obesity enhances renal Arg-II level and that genetic ablation of Arg-II protects mice from the renal pathological changes in diet-induced obesity.

\section{Role of Arg-II in Diabetic Kidney Disease}

It is well known that kidney expresses abundant Arg-II (Morris et al., 2011). The physiological role of Arg-II in renal function is not known. Some studies demonstrate that Arg-II may participate in pathogenesis of renal diseases associated with type 1 diabetes in mouse models. For example, pharmacological inhibition of Arg-II in spontaneously diabetic Ins $2^{\text {Akita }}$ mice or streptozotocin (STZ)-induced diabetic Dilute Brown Agouti (DBA) mice is able to attenuate renal injuries, including albuminuria and kidney macrophage infiltration (Morris et al., 2011; You et al., 2013). Also, mice with genetic Arg-II deficiency 

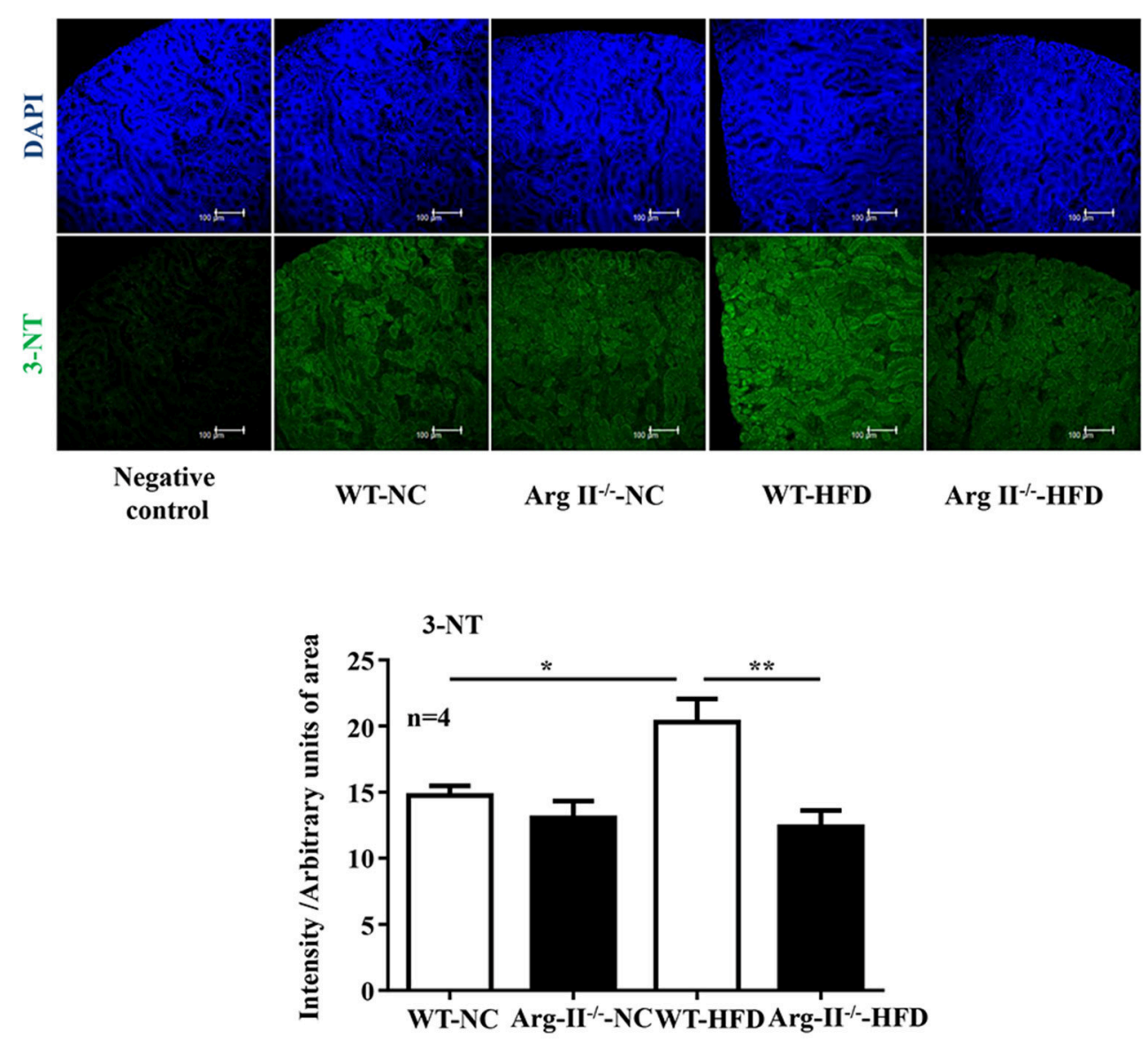

FIGURE 6 | Arg-II deficiency prevents obesity-associated increase in renal peroxynitrite formation. Confocal immunofluorescence staining of 3-NT (green) and DAPI (blue) in the kidney of WT and Arg- II-/- mice fed either normal chow (NC) or high fat diet (HFD). Representative images of individual staining and merged images are shown $(n=4)$. Quantifications of 3-NT signals are shown in the bar graphs. ${ }^{\star} p<0.05,{ }^{* \star} p<0.01$

are protected from renal injuries in STZ-induced diabetes (Morris et al., 2011). These studies suggest an important role of Arg-II in type 1 diabetic renal disease. However, another group demonstrates opposite effects, i.e., Arg-II deficiency accelerates some of the STZ-induced renal damages in mice (Romero et al., 2013). The discrepancy between the two studies is not clear. In accordance with the study in type 1 diabetic mouse model in which renal arginase activity is increased (Morris et al., 2011), we also demonstrate in the current study an increase in Arg-II levels and arginase activity in kidney of the obesity-associated type 2 mouse model. Since Arg-I is not detectable in the kidney, the increase in arginase activity in kidney is solely attributable to Arg-II.

\section{Arg-II Is Mainly Localized in the Straight Segment of Proximal Tubules}

In line with the observation in kidney, we have previously shown that in the same diet-induced obesity mouse model, Arg-II is upregulated in other cells such as macrophages and vascular endothelial cells (Yu et al., 2014). The increase in Arg-II in macrophages under HFD feeding promotes pro-inflammatory responses, contributing to obesity-associated glucose intolerance, insulin resistance, and nonalcoholic fatty liver diseases (NAFLD) as well as atherogenesis (Ming et al., 2012; Liu et al., 2016). In kidney, we demonstrate in this study that Arg-II is mainly expressed in the S3 straight segment of the proximal tubules as demonstrated by expression in the B0AT3 positive cells. In the glomeruli, however, no ArgII signal could be detected by immunofluorescence analysis. At this stage, the mechanisms of upregulation of Arg-II in kidney are not known. In the vascular cells, Arg-II upregulation involves activation of mTOR/S6K1 and p38mapk signaling pathways (Yepuri et al., 2012; Xiong et al., 2013, 2014; Wu et al., 2015). Whether these mechanisms are also involved in the obesity-associated renal injury remains to be investigated.

\section{Arg-II Causes Renal Oxidative Stress through NOS-Uncoupling in Obesity}

It has been shown that Arg-II upregulation in blood vessels of obesity mice causes endothelial dysfunction and eNOSuncoupling, leading to decrease in NO production and 


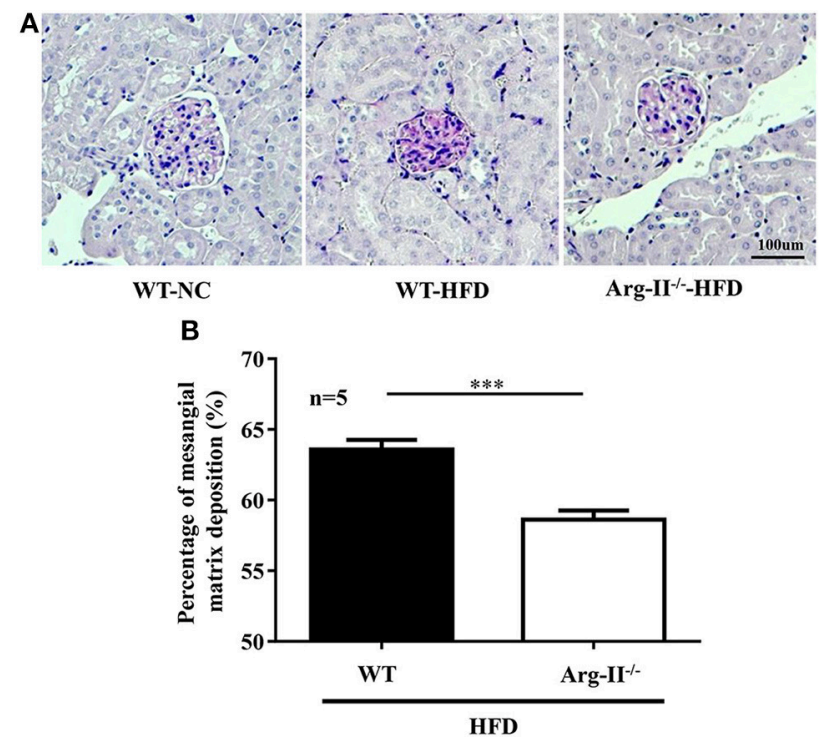

FIGURE 7 | Arg-Il-deficiency protects against mesangial expansion in obesity. (A) PAS staining (magenta) of paraffin-embedded kidney sections shows decreased mesangial expansion in Arg- $\mathrm{II}^{-/}-$mice as compared to WT mice on HFD. Nuclei were counter stained by hematoxylin (blue). Kidney from a WT mice on NC diet was used as a control. (B) Bar graphs show

quantification of mesangial expansion. $n=5,{ }^{\star \star \star} p<0.001$ vs. WT-HFD group.

increase in superoxide radical anion levels (Yu et al., 2014). Importantly, eNOS-uncoupling has been reported to contribute to the pathogenesis of diabetic nephropathy (Prabhakar et al., 2007). In line with these findings, we demonstrate increased expression levels and activity of Arg-II in kidney of dietinduced obesity mice, which causes renal oxidative stress due to NOS-uncoupling, since the NOS inhibitor, L-NAME, reduces ROS levels as assessed by DHE staining. NO can react with superoxide radical anion to produce peroxynitrite which is able to cause nitration of tyrosine residues of proteins, i.e., nitrosative stress (Förstermann and Sessa, 2012). The modification of proteins at tyrosine residues often leads to functional decline of enzyme activity. It has been shown that the mitochondrial antioxidant enzyme manganese superoxide dismutase (MnSOD) is modified by the nitrosative stress and contains nitrotyrosine in angiotensin II-induced hypertensive rat kidney, which may facilitate the oxidative/nitrosative stress and renal injury (Xu et al., 2006). Various studies provide evidence for the important role of oxidative/nitrosative stress in renal injury and disease progression (Ratliff et al., 2016; Sharma, 2016). In support of this finding, the oxidative/nitrosative stress as assessed by increased levels of superoxide radical anion and 3-NT in kidney of obesity mice is prevented in $\mathrm{Arg}-\mathrm{II}^{-/-}$animals despite no changes in body weight and kidney weight/body weight ratio. It is to mention that myeloperoxidase and other peroxidases are also involved in nitrotyrosine formation (Gaut et al., 2002). Functional roles of these enzymes in renal nitrosative stress in the mouse model could not be excluded and require further investigation.

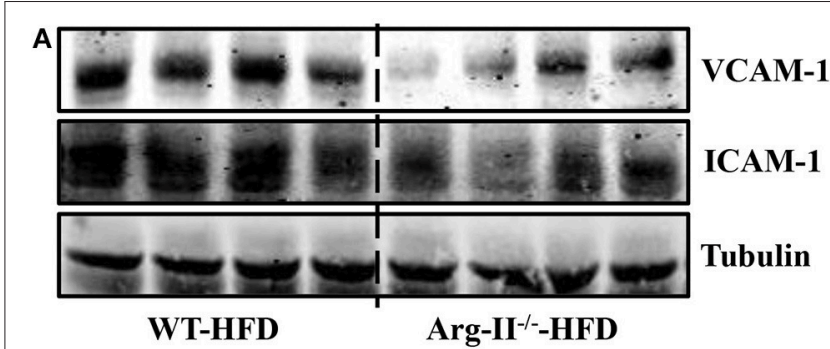

B

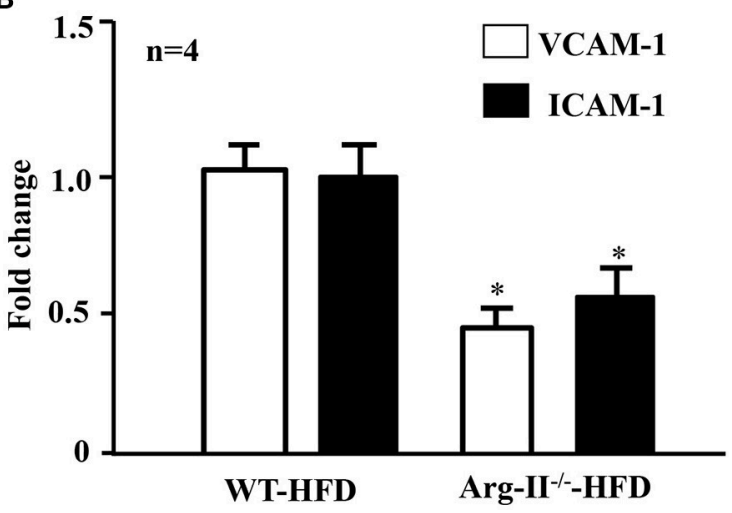

FIGURE 8 | Arg-II-deficiency reduces adhesion molecule levels in the kidney in obesity. (A) Immunoblotting analysis of VCAM-1 and ICAM-I expression in the kidney of WT and Arg- $\|^{-/-}$mice fed HFD. (B) Bar graphs show quantification of VCAM-1 and ICAM-I expression. $n=4,{ }^{*} p<0.05$ between indicated groups.

Oxidative stress derived from eNOS-uncoupling caused by Arg-II is causatively involved in adhesion molecule expression in the endothelial cells (Yepuri et al., 2012). In line with this observation, our present study demonstrates that Arg-II and adhesion molecule expression in kidney are upregulated in obesity and that Arg-II deficiency reduces oxidative stress, decreases VCAM-1 and ICAM-1 levels in kidney of the obesity mouse model. Together, these results suggest that Arg-II indeed plays an important role in obesity-associated renal damage involving NOS-uncoupling. Since kidney expresses eNOS, iNOS, and nNOS (Prabhakar et al., 2007) and decreased renal NO bioavailability is linked to diabetic renal disease (Erdely et al., 2004; Prabhakar, 2005), the question whether each of these isoenzymes are uncoupled in kidney of obesity mice remains to be investigated. Another important question which remains to be investigated is how the proximal tubule straight segments interact with glomerulus cells and participate in diabetic renal injury.

\section{Role of Arg-II in Renal Lipid Accumulation in Obesity}

It is important to point out that the $\mathrm{Arg}-\mathrm{II}^{-/-}$mice are protected from whole body glucose intolerance with improved insulin sensitivity and less NAFLD on HFD (Liu et al., 2016). Similar to the liver, we also found a decreased renal lipid accumulation in kidney as shown in our current study. The renal lipid accumulation in type 2 diabetes is 
well described in humans and animal models, which is considered as an important contributor to progression of diabetic nephropathy in obesity (Jiang et al., 2005). The underlying mechanisms are attributable to the increased expression of the transcriptional factors SREBP-1c, the master regulator of lipogenesis and suppressed renal lipolysis (Jiang et al., 2005; Kume et al., 2007). The results of our current study suggest an important role of Arg-II in obesityassociated renal diseases. It remains to be investigated how important the renal Arg-II is in the development of obesityassociated kidney damage. For this purpose, renal specific Arg-II knockout mouse model shall be generated in the future.

\section{CONCLUSIONS}

We demonstrate that Arg-II deficiency in mouse protects against obesity-associated renal alterations. The results suggest that targeting Arg-II may represent a promising therapeutic strategy for diabetic nephropathy.

\section{REFERENCES}

Ash, D. E. (2004). Structure and function of arginases. J. Nutr. 134, 2760S-2764S.

Cheng, H., and Harris, R. C. (2014). Renal endothelial dysfunction in diabetic nephropathy. Cardiovasc. Hematol. Disord Drug Targets 14, 22-33. doi: 10. 2174/1871529X14666140401110841

Cheng, H., Wang, H., Fan, X., Paueksakon, P., and Harris, R. C. (2012). Improvement of endothelial nitric oxide synthase activity retards the progression of diabetic nephropathy in $\mathrm{db} / \mathrm{db}$ mice. Kidney Int. 82, 1176-1183. doi: $10.1038 / \mathrm{ki} .2012 .248$

Collaboration, N. C. D. R. F. (2016). Worldwide trends in diabetes since 1980: a pooled analysis of 751 population-based studies with 4.4 million participants. Lancet 387, 1513-1530. doi: 10.1016/S0140-6736(16)00618-8

Collins, A. J., Foley, R. N., Herzog, C., Chavers, B., Gilbertson, D., Herzog, C., et al. (2013). US renal data system 2012 annual data report. Am. J. Kidney Dis. 61, e1-476. doi: 10.1053/j.ajkd.2012.11.031

Crombez, E. A., and Cederbaum, S. D. (2005). Hyperargininemia due to liver arginase deficiency. Mol. Genet. Metab. 84, 243-251. doi: 10.1016/j.ymgme. 2004.11.004

Cui, W., Maimaitiyiming, H., Qi, X., Norman, H., and Wang, S. (2013). Thrombospondin 1 mediates renal dysfunction in a mouse model of high-fat diet-induced obesity. Am. J. Physiol. Renal Physiol. 305, F871-F880. doi: 10. 1152/ajprenal.00209.2013

Declèves, A. E., Mathew, A. V., Cunard, R., and Sharma, K. (2011). AMPK mediates the initiation of kidney disease induced by a high-fat diet. J. Am. Soc. Nephrol. 22, 1846-1855. doi: 10.1681/ASN.2011010026

Deji, N., Kume, S., Araki, S., Soumura, M., Sugimoto, T., and Isshiki, K., et al. (2009). Structural and functional changes in the kidneys of high-fat dietinduced obese mice. Am. J. Physiol. Renal Physiol. 296, F118-F126. doi: 10. 1152/ajprenal.00110.2008

DeRubertis, F. R., Craven, P. A., Melhem, M. F., and Salah, E. M. (2004). Attenuation of renal injury in $\mathrm{db} / \mathrm{db}$ mice overexpressing superoxide dismutase: evidence for reduced superoxide-nitric oxide interaction. Diabetes 53, 762-768. doi: 10.2337/diabetes.53.3.762

Erdely, A., Freshour, G., Maddox, D. A., Olson, J. L., Samsell, L., and Baylis, C. (2004). Renal disease in rats with type 2 diabetes is associated with decreased renal nitric oxide production. Diabetologia 47, 1672-1676. doi: 10.1007/s00125004-1509-1

Fioretto, P., Caramori, M. L., and Mauer, M. (2008). The kidney in diabetes: dynamic pathways of injury and repair. The Camillo Golgi Lecture 2007. Diabetologia 51, 1347-1355. doi: 10.1007/s00125-008-1051-7

\section{AUTHOR CONTRIBUTIONS}

$\mathrm{JH}, \mathrm{AR}$, and $\mathrm{YX}$ : acquisition, analysis, or interpretation of data for the work; preparation of figures; critically revised the manuscript for important intellectual content. XM and ZY: design of the work; analyzed and interpreted primary research papers, drafted the manuscript and critically revised the manuscript for important intellectual content. FV: generated and validated antibody against B0AT3 and critically revised the manuscript for important intellectual content. JM: interpreted primary research papers, critically revised the manuscript for important intellectual content. All authors agree to be accountable for the content of the work.

\section{FUNDING}

This work was supported by the Swiss National Science Foundation (31003A_159582/1), Swiss Heart Foundation, and National Centre of Competence in Research Program (NCCRKidney.CH).

Förstermann, U., and Sessa, W. C. (2012). Nitric oxide synthases: regulation and function. Eur. Heart J. 33, 829-837. doi: 10.1093/eurheartj/ ehr304

Gaut, J. P., Byun, J., Tran, H. D., Lauber, W. M., Carroll, J. A., and Hotchkiss, R. S., et al. (2002). Myeloperoxidase produces nitrating oxidants in vivo. J. Clin. Invest. 109, 1311-1319. doi: 10.1172/JCI0215021

Goligorsky, M. S., Chen, J., and Brodsky, S. (2001). Workshop: endothelial cell dysfunction leading to diabetic nephropathy: focus on nitric oxide. Hypertension 37, 744-748. doi: 10.1161/01.HYP.37.2.744

Gotoh, T., Araki, M., and Mori, M. (1997). Chromosomal localization of the human arginase II gene and tissue distribution of its mRNA. Biochem. Biophys. Res. Commun. 233, 487-491. doi: 10.1006/bbrc.1997.6473

Jiang, T., Wang, Z., Proctor, G., Moskowitz, S., Liebman, S. E., and Rogers, T. et al. (2005). Diet-induced obesity in C57BL/6J mice causes increased renal lipid accumulation and glomerulosclerosis via a sterol regulatory element-binding protein-1c-dependent pathway. J. Biol. Chem. 280, 32317-32325. doi: 10.1074/ jbc.M500801200

Kume, S., Uzu, T., Araki, S., Sugimoto, T., Isshiki, K., and Chin-Kanasaki, M., et al (2007). Role of altered renal lipid metabolism in the development of renal injury induced by a high-fat diet. J. Am. Soc. Nephrol. 18, 2715-2723. doi: 10.1681/ ASN.2007010089

Lal, M. A., Körner, A., Matsuo, Y., Zelenin, S., Cheng, S. X., and Jaremko, G., et al. (2000). Combined antioxidant and COMT inhibitor treatment reverses renal abnormalities in diabetic rats. Diabetes 49, 1381-1389. doi: 10.2337/diabetes. 49.8.1381

Li, H., and Förstermann, U. (2013). Uncoupling of endothelial NO synthase in atherosclerosis and vascular disease. Curr. Opin. Pharmacol. 13, 161-167. doi: 10.1016/j.coph.2013.01.006

Liu, C., Rajapakse, A. G., Riedo, E., Fellay, B., Bernhard, M. C., and Montani, J. P., et al. (2016). Targeting arginase-II protects mice from high-fat-diet-induced hepatic steatosis through suppression of macrophage inflammation. Sci. Rep. 6:20405. doi: 10.1038/srep20405

Menini, S., Amadio, L., Oddi, G., Ricci, C., Pesce, C., and Pugliese, F., et al. (2006). Deletion of p66Shc longevity gene protects against experimental diabetic glomerulopathy by preventing diabetes-induced oxidative stress. Diabetes 55, 1642-1650. doi: $10.2337 / \mathrm{db} 05-1477$

Ming, X. F., Rajapakse, A. G., Yepuri, G., Xiong, Y., Carvas, J. M., and Ruffieux, J., et al. (2012). Arginase II Promotes macrophage inflammatory responses through mitochondrial reactive oxygen species, contributing to insulin resistance and atherogenesis. J. Am. Heart Assoc. 1:e000992. doi: 10. 1161/JAHA.112.000992 
Morris, S. M. Jr., Gao, T., Cooper, T. K., Kepka-Lenhart, D., and Awad, A. S. (2011). Arginase-2 mediates diabetic renal injury. Diabetes 60, 3015-3022. doi: 10.2337/ db11-0901

Nakagawa, T., Tanabe, K., Croker, B. P., Johnson, R. J., Grant, M. B., Kosugi, T., et al. (2011). Endothelial dysfunction as a potential contributor in diabetic nephropathy. Nat. Rev. Nephrol. 7, 36-44. doi: 10.1038/nrneph.2010.152

Prabhakar, S. S. (2005). Pathogenic role of nitric oxide alterations in diabetic nephropathy. Curr. Diab. Rep. 5, 449-454. doi: 10.1007/s11892-005-0054-8

Prabhakar, S., Starnes, J., Shi, S., Lonis, B., and Tran, R. (2007). Diabetic nephropathy is associated with oxidative stress and decreased renal nitric oxide production. J. Am. Soc. Nephrol. 18, 2945-2952. doi: 10.1681/ASN.2006080895

Rajapakse, A. G., Yepuri, G., Carvas, J. M., Stein, S., Matter, C. M., and Scerri, I., et al. (2011). Hyperactive S6K1 mediates oxidative stress and endothelial dysfunction in aging: inhibition by resveratrol. PLOS ONE 6:e19237. doi: 10 . 1371/journal.pone.0019237

Ramírez-Zacarías, J. L., Castro-Muñozledo, F., and Kuri-Harcuch, W. (1992). Quantitation of adipose conversion and triglycerides by staining intracytoplasmic lipids with Oil red O. Histochemistry 97, 493-497. doi: 10. 1007/BF00316069

Ratliff, B. B., Abdulmahdi, W., Pawar, R., and Wolin, M. S. (2016). Oxidant mechanisms in renal injury and disease. Antioxid. Redox Signal. 25, 119-146. doi: 10.1089/ars.2016.6665

Romeo, E., Dave, M. H., Bacic, D., Ristic, Z., Camargo, S. M., Loffing, J., et al. (2006). Luminal kidney and intestine SLC6 amino acid transporters of B0ATcluster and their tissue distribution in Mus musculus. Am. J. Physiol. Renal Physiol. 290, F376-F383. doi: 10.1152/ajprenal.00286.2005

Romero, M. J., Yao, L., Sridhar, S., Bhatta, A., Dou, H., and Ramesh, G., et al. (2013). 1-citrulline protects from kidney damage in type 1 diabetic mice. Front. Immunol. 4:480. doi: 10.3389/fimmu.2013.00480

Sharma, K. (2016). Obesity and diabetic kidney disease: role of oxidant stress and redox balance. Antioxid. Redox Signal. 25, 208-216. doi: 10.1089/ars.2016.6696

Shi, O., Morris, S. M. Jr., Zoghbi, H., Porter, C. W., and O’Brien, W. E. (2001). Generation of a mouse model for arginase II deficiency by targeted disruption of the arginase II gene. Mol. Cell. Biol. 21, 811-813. doi: 10.1128/MCB.21.3.811813.2001

Sparkes, R. S., Dizikes, G. J., Klisak, I., Grody, W. W., Mohandas, T., and Heinzmann, C., et al. (1986). The gene for human liver arginase (ARG1) is assigned to chromosome band 6q23. Am. J. Hum. Genet. 39, 186-193.

Toque, H. A., Nunes, K. P., Yao, L., Xu, Z., Kondrikov, D., Su, Y., et al. (2013). Akita spontaneously type 1 diabetic mice exhibit elevated vascular arginase and impaired vascular endothelial and nitrergic function. PLoS ONE 8:e72277. doi: 10.1371/journal.pone.0072277

Tsang, J. P., Poon, W. L., Luk, H. M., Fung, C. W., Ching, C. K., Mak, C. M., et al. (2012). Arginase deficiency with new phenotype and a novel mutation: contemporary summary. Pediatr. Neurol. 47, 263-269. doi: 10.1016/ j.pediatrneurol.2012.06.012

Wang, C. H., Li, F., Hiller, S., Kim, H. S., Maeda, N., Smithies, O., et al. (2011). A modest decrease in endothelial NOS in mice comparable to that associated with human NOS3 variants exacerbates diabetic nephropathy. Proc. Natl. Acad. Sci. U.S.A. 108, 2070-2075. doi: 10.1073/pnas.1018766108
Wu, Z., Yu, Y., Liu, C., Xiong, Y., Montani, J. P., Yang, Z., et al. (2015). Role of p38 mitogen-activated protein kinase in vascular endothelial aging: interaction with Arginase-II and S6K1 signaling pathway. Aging (Albany. NY). 7, 70-81. doi: 10. 18632/aging.100722

Xiong, Y., Yepuri, G., Forbiteh, M., Yu, Y., Montani, J. P., Yang, Z., et al. (2014). ARG2 impairs endothelial autophagy through regulation of MTOR and PRKAA/AMPK signaling in advanced atherosclerosis. Autophagy 10, 2223-2238. doi: 10.4161/15548627.2014.981789

Xiong, Y., Yu, Y., Montani, J. P., Yang, Z., and Ming, X. F. (2013). Arginase-II induces vascular smooth muscle cell senescence and apoptosis through p66Shc and p53 independently of its l-arginine ureahydrolase activity: implications for atherosclerotic plaque vulnerability. J. Am. Heart Assoc. 2:e000096. doi: 10. 1161/JAHA.113.000096

Xu, S., Ying, J., Jiang, B., Guo, W., Adachi, T., Sharov, V., et al. (2006). Detection of sequence-specific tyrosine nitration of manganese SOD and SERCA in cardiovascular disease and aging. Am. J. Physiol. Heart Circ. Physiol. 290, H2220-H2227. doi: 10.1152/ajpheart.01293.2005

Yang, Z., and Ming, X. F. (2013). Arginase: the emerging therapeutic target for vascular oxidative stress and inflammation. Front. Immunol. 4:149. doi: 10 3389/fimmu.2013.00149

Yang, Z., and Ming, X. F. (2014). Functions of arginase isoforms in macrophage inflammatory responses: impact on cardiovascular diseases and metabolic disorders. Front. Immunol. 5:533. doi: 10.3389/fimmu.2014.00533

Yepuri, G., Velagapudi, S., Xiong, Y. Y., Rajapakse, A. G., Montani, J. P., Ming, X. F., et al. (2012). Positive crosstalk between arginase-II and S6K1 in vascular endothelial inflammation and aging. Aging Cell 11, 1005-1016. doi: 10.1111/ acel.12001

You, H., Gao, T., Cooper, T. K., Morris, S. M. Jr., and Awad, A. S. (2013). Arginase inhibition mediates renal tissue protection in diabetic nephropathy by a nitric oxide synthase 3-dependent mechanism. Kidney Int. 84, 1189-1197. doi: 10. 1038/ki.2013.215

You, H., Gao, T., Cooper, T. K., Morris, S. M. Jr., and Awad, A. S. (2014). Diabetic nephropathy is resistant to oral L-arginine or L-citrulline supplementation. Am. J. Physiol. Renal Physiol. 307, F1292-F1301. doi: 10.1152/ajprenal.00176.2014

Yu, Y., Rajapakse, A. G., Montani, J. P., Yang, Z., and Ming, X. F. (2014). p38 mitogen-activated protein kinase is involved in arginase-II-mediated eNOSUncoupling in Obesity. Cardiovasc. Diabetol. 13:113. doi: 10.1186/s12933-0140113-z

Conflict of Interest Statement: The authors declare that the research was conducted in the absence of any commercial or financial relationships that could be construed as a potential conflict of interest.

Copyright (c) 2016 Huang, Rajapakse, Xiong, Montani, Verrey, Ming and Yang. This is an open-access article distributed under the terms of the Creative Commons Attribution License (CC BY). The use, distribution or reproduction in other forums is permitted, provided the original author(s) or licensor are credited and that the original publication in this journal is cited, in accordance with accepted academic practice. No use, distribution or reproduction is permitted which does not comply with these terms. 